\title{
MEASURES ON THE PRODUCT OF COMPACT SPACES
}

\author{
IGNACIO VILLANUEVA
}

\begin{abstract}
If $K$ is an uncountable metrizable compact space, we prove a "factorization" result for a wide variety of vector valued Borel measures $\mu$ defined on $K^{n}$. This result essentially says that for every such measure $\mu$ there exists a measure $\mu^{\prime}$ defined on $K$ such that the measure $\mu$ of a product $A_{1} \times \cdots \times A_{n}$ of Borel sets of $K$ equals the measure $\mu^{\prime}$ of the intersection $A_{1}^{\prime} \cap \cdots \cap A_{n}^{\prime}$, where the $A_{i}^{\prime}$ 's are certain transforms of the $A_{i}$ 's.
\end{abstract}

\section{INTRODUCTION AND NOTATION}

In [6] we proved, among others, the following result.

Proposition 1.1. Let $K$ be a metrizable uncountable compact space, $n \in \mathbb{N}$. Then there exist isometric injections $A_{m}: C(K) \hookrightarrow C(K)(1 \leq m \leq n)$ so that, for every Banach space $X$ and every operator $T: \widehat{\otimes}_{\epsilon}^{n} C(K) \longrightarrow X$ there exists an operator $B: C(K) \longrightarrow X$ such that,

$$
T\left(f_{1} \otimes \cdots \otimes f_{n}\right)=B\left(\prod_{m=1}^{n} A_{m}\left(f_{m}\right)\right) .
$$

The purpose of this note is to prove two measure theoretic results related with, and independent of, Proposition 1.1 (Theorems 2.1 and 2.2 below). The ideas we use are based on ideas in [5] and [6], and ultimately they rely on the theory of averaging operators developed by Pełcyński in [3].

First we explain our notation and some basic facts we use. We write $I d_{X}$ for the identity operator on a Banach space $X$. $\mathbb{1}_{K}$ will stand for the function constantly equal 1 defined on $K$. Given two compact spaces $K$ and $S$ and a continuous function $\varphi: K \longrightarrow S$, we can always define the linear operator $\varphi^{o}: C(S) \longrightarrow C(K)$ of norm one given by $\varphi^{o}(f)(t)=$ $f(\varphi(t))$. If $\varphi$ is injective, then $\varphi^{o}$ is a quotient, and if $\varphi$ is onto, then $\varphi^{o}$ is an injective isometry. Suppose $\varphi$ is injective (resp. onto). If there exists $u: C(K) \longrightarrow C(S)$ such that $\varphi \circ u=I d_{C(K)}\left(\right.$ resp. $\left.u \circ \varphi=I d_{C(S)}\right)$ then we say that $u$ is an extension operator (resp. averaging operator) for $\varphi$. Moreover, if $\|u\|=1$ and $u\left(\mathbb{1}_{K}\right)=\mathbb{1}_{S}$, then $u$ is said to be regular

1991 Mathematics Subject Classification. 28A45, 46G10.

Key words and phrases. Uncountable metrizable compact space, vector valued measures.

Partially supported by DGICYT grant PB97-0240. 
(see [3] for information concerning extension and averaging operators). We write $\otimes^{n} X$ for the tensor product of $X$ with itself $n$ times and $\widehat{\otimes}_{\epsilon}^{n} X$ for the completion of $\otimes^{n} X$ under the injective norm (see, for example, [2] for general references about the injective tensor product). If $K$ is a compact, we write $K^{n}$ for the compact set $K \times \stackrel{(n)}{\cdots} \times K$. We recall that $\widehat{\otimes}_{\epsilon}^{n} C(K)$ is isometrically isomorphic to $C\left(K^{n}\right)$, the isomorphism $\vartheta: \widehat{\otimes}_{\epsilon}^{n} C(K) \longrightarrow C\left(K^{n}\right)$ verifying $\vartheta\left(f_{1} \otimes \cdots \otimes f_{n}\right)\left(t_{1}, \ldots, t_{n}\right)=\prod_{m=1}^{n} f_{m}\left(t_{m}\right)$. Thus we identify $\widehat{\otimes}_{\epsilon}^{n} C(K)$ and $C\left(K^{n}\right)$. Given two Banach spaces $X$ and $Y$, we write $\mathcal{L}(X ; Y)$ for the space of linear bounded operators from $X$ into $Y$.

Given a compact space $K$, we write $\Sigma_{K}$ for the $\sigma$-algebra of the Borel sets of $K$. Given a $\sigma$-algebra $\Sigma$ and a Banach space $X$, we write $S(\Sigma, X)$ for the vector space of the $X$-valued $\Sigma$-measurable simple functions, and we write $B(\Sigma, X)$ for the completion of $S(\Sigma, X)$ under the supremum norm. If $X=\mathbb{K}$ then we omit it. There is an isometric isomorphism between $B(\Sigma)^{*}$ and $b a(\Sigma)$, the space of scalar bounded finitely additive measures: $\alpha \in B(\Sigma)^{*}$ and $\mu \in b a(\Sigma)$ are associated through this isometry if $\alpha\left(\chi_{A}\right)=$ $\mu(A)$ for every $A \in \Sigma$. Similarly, the space $b a(\Sigma ; X)$ of the bounded finitely additive measures defined on $\Sigma$ with valued in $X$ is isometrically isomorphic to $\mathcal{L}(B(\Sigma) ; X)$ (see $[2$, Section VI.1]).

It is a simple consequence of the Riesz representation theorem that $B\left(\Sigma_{K}\right)$ is isometrically contained in $C(K)^{* *}$. Given an operator $T: C(K) \longrightarrow X$, we define its extension $\widetilde{T}=T_{\left.\right|_{B\left(\Sigma_{K}\right)} ^{* *}}^{*}: B\left(\Sigma_{K}\right) \longrightarrow X^{* *}$. It is well known that the simplest way to define the representing measure $\mu: \Sigma_{K} \longrightarrow X^{* *}$ of $T$ is to define $\mu(A)=\widetilde{T}\left(\chi_{A}\right)$ (see [2, Chapter VI] for a very nice presentation of this and related facts).

\section{The Results}

Let $\varphi: S \longrightarrow K$ be a continuous mapping and let $\varphi^{o}: C(K) \longrightarrow C(S)$ be the associated mapping given by $\varphi^{o}(f)(t)=f(\varphi(t))$. Let us see that its extension $\widetilde{\varphi^{o}}: B\left(\Sigma_{K}\right) \longrightarrow C(S)^{* *}$ actually takes values in $B\left(\Sigma_{S}\right)$, and that $\widetilde{\varphi^{o}}(g)(t)=g(\varphi(t))$. To prove this, let $\theta: B\left(\Sigma_{K}\right) \longrightarrow B\left(\Sigma_{S}\right)$ be the mapping given by $\theta(g)(t)=g(\varphi(t))$. Since $\theta_{\left.\right|_{C(K)}}=\varphi^{o}$, it suffices to see that $\theta$ is $\sigma\left(B\left(\Sigma_{K}\right), C(K)^{*}\right)-\sigma\left(B\left(\Sigma_{S}\right), C(S)^{*}\right)$ continuous. So, let $\left(g_{\alpha}\right) \subset B\left(\Sigma_{K}\right)$ be a bounded net $\sigma\left(B\left(\Sigma_{K}\right), C(K)^{*}\right)$ convergent to $g \in B\left(\Sigma_{K}\right)$ and let $\mu \in$ $C(S)^{*}$. We can define $\mu^{\prime} \in C(K)^{*}$ by $\mu^{\prime}(A)=\mu\left(\varphi^{-1}(A)\right)$. Then, for every $g \in B\left(\Sigma_{K}\right)$

$$
\int_{K} g(t) d \mu^{\prime}(t)=\int_{S} g(\varphi(s)) d \mu(s)
$$

(in fact, $\mu^{\prime}=\varphi^{o *}(\mu)$ ). Therefore,

$$
\begin{aligned}
\left\langle\theta\left(g_{\alpha}\right), \mu\right\rangle= & \int_{S} g_{\alpha}(\varphi(s)) d \mu(s)=\int_{K} g_{\alpha}(t) d \mu^{\prime}(t)=\left\langle g_{\alpha}, \mu^{\prime}\right\rangle \longrightarrow\left\langle g, \mu^{\prime}\right\rangle= \\
& =\int_{K} g(t) d \mu^{\prime}(t)=\int_{S} g(\varphi(s)) d \mu(s)=\langle\theta(g), \mu\rangle .
\end{aligned}
$$


So, $\theta\left(g_{\alpha}\right)$ converges $\sigma\left(B\left(\Sigma_{S}\right), C(S)^{*}\right)$ to $\theta(g)$ and hence $\widetilde{\varphi^{o}}=\theta$. Note that $\varphi^{o}$ and $\widetilde{\varphi^{o}}$ are linear and multiplicative.

We will also use the following fact: if $\Sigma_{1}$ and $\Sigma_{2}$ are two $\sigma$-algebras (or just algebras) and $\psi: B\left(\Sigma_{1}\right) \longrightarrow B\left(\Sigma_{2}\right)$ is a linear and multiplicative operator, then it takes characteristic functions into characteristic functions (because $\left.\chi_{A}=\chi_{A}^{2}\right)$.

Let us also recall that, if $K$ is metrizable, then $\Sigma_{K} \otimes \stackrel{(n)}{\cdots} \otimes \Sigma_{K}=\Sigma_{K^{n}}$, where $\Sigma_{K} \otimes \stackrel{(n)}{\cdots} \otimes \Sigma_{K}$ denotes the product $\sigma$-algebra, i.e., the smallest $\sigma$ algebra that contains the rectangles $\left(A_{1} \times \cdots \times A_{n}\right) \subset K^{n}$, with $A_{i} \in \Sigma_{K}$ $(1 \leq i \leq n)($ see $[1,7.6 .2$ and 7.1.12]).

Following the ideas of [2, Example VIII.1.6] it can be proved that if $X$ is a Banach space and $\Sigma$ is a $\sigma$-algebra, then $B(\Sigma) \hat{\otimes}_{\epsilon} X$ is isometrically isomorphic to $B(\Sigma, X)$. Hence, $B\left(\Sigma_{K}\right) \hat{\otimes}_{\epsilon} B\left(\Sigma_{K}\right)$ is isometrically isomorphic to $B\left(\Sigma_{K}, B\left(\Sigma_{K}\right)\right)$, the isomorphism $\psi$ verifying $\psi(f \otimes g)(s)(t)=f(s) g(t)$. Moreover, there is an isometric injection $i: B\left(\Sigma_{K}, B\left(\Sigma_{K}\right)\right) \hookrightarrow B\left(\Sigma_{K} \otimes \Sigma_{K}\right)$ given by $i(f)(s, t)=f(s)(t)$.

Therefore, using the fact that the $\epsilon$ topology respects isometric injections, we get that there is a canonical injective isometry $i^{\prime}: \widehat{\otimes}_{\epsilon}^{n} B\left(\Sigma_{K}\right) \hookrightarrow B\left(\Sigma_{K^{n}}\right)$.

We can present now the first of our results.

Theorem 2.1. Let $K$ be a metrizable uncountable compact space, $n \in \mathbb{N}$. Then there exist $n$ different linear and multiplicative isometric inclusions $I_{m}: B\left(\Sigma_{K}\right) \hookrightarrow B\left(\Sigma_{K}\right),(1 \leq m \leq n)$ (taking characteristic functions to characteristic functions) such that, for every Banach space $X$ and every operator $T: \widehat{\otimes}_{\epsilon}^{n} C(K) \longrightarrow X$ with associated measure $\mu: \Sigma_{K^{n}} \longrightarrow X^{* *}$ there exists an operator $B: C(K) \longrightarrow X$ with representing measure $\mu_{B}:$ $\Sigma_{K} \longrightarrow X^{* *}$ so that

$$
\mu\left(A_{1} \times \cdots \times A_{n}\right)=\mu_{B}\left(\bigcap_{m=1}^{n} A_{m}^{\prime}\right),
$$

where $I_{m}\left(\chi_{A_{m}}\right)=\chi_{A_{m}^{\prime}}$. Moreover, $\mu$ is $X$-valued (equivalently regular or countably additive) if and only if $\mu_{B}$ is $X$-valued.

Proof. Let $i_{m}: B\left(\Sigma_{K}\right) \hookrightarrow B\left(\Sigma_{K^{n}}\right)$ be the isometric inclusion given by $i_{m}(g)\left(t_{1}, \ldots, t_{n}\right)=g\left(t_{m}\right)$. Let $\Delta \subset K$ be a subspace homeomorphic to the Cantor set. Let $\varphi: \Delta \longrightarrow K^{n}$ be an onto continuous mapping admitting an averaging operator $u: C(\Delta) \longrightarrow C\left(K^{n}\right)$ (the existence of one such $\varphi$ and $u$ follows from [3, Theorem 5.6]). Let $\widetilde{\varphi^{o}}: B\left(\Sigma_{K^{n}}\right) \hookrightarrow B\left(\Sigma_{\Delta}\right)$ be the operator associated to $\varphi$ (see the comments before this theorem). Then $I d_{C\left(K^{n}\right)}=u \circ \varphi^{o}$, so

$$
I d_{B\left(\Sigma_{K^{n}}\right)}=\widetilde{u \circ \varphi^{o}}=\left(u \circ \varphi^{o}\right)_{\left.\right|_{B\left(\Sigma_{K^{n}}\right)} ^{* *}}=u^{* *} \circ\left(\varphi^{o}\right)_{\left.\right|_{B\left(\Sigma_{K^{n}}\right)} ^{* *}}^{*}=\widetilde{u} \odot \widetilde{\varphi^{o}}
$$


because $\widetilde{\varphi^{o}}\left(B\left(\Sigma_{K^{n}}\right) \subset B\left(\Sigma_{\Delta}\right)\right.$. Since $\widetilde{\varphi^{o}}(g)(t)=g(\varphi(t))$ (see the remarks before the theorem), for every $f, g \in B\left(\Sigma_{K^{n}}\right), \widetilde{\varphi^{o}}(g) \widetilde{\varphi^{o}}(f)=\widetilde{\varphi^{o}}(g f)$ and, hence, $\widetilde{u}\left(\widetilde{\varphi^{o}}(g) \widetilde{\varphi^{o}}(f)\right)=g f$.

Now we consider the injective isometry

$$
\bar{E}: B\left(\Sigma_{\Delta}\right) \hookrightarrow B\left(\Sigma_{K}\right)
$$

defined by

$$
\bar{E}(g)(t)=\left\{\begin{array}{lll}
g(t) & \text { if } & t \in \Delta \\
0 & \text { if } & t \notin \Delta
\end{array}\right.
$$

Note that, if $E: C(\Delta) \longrightarrow C(K)$ is a Borsuk-Dugundji extension operator (see, for example, [4, Theorem 21.1.4]), we can not consider $\widetilde{E}$ here, because we do not know whether $\widetilde{E}\left(B\left(\Sigma_{\Delta}\right)\right) \subset B\left(\Sigma_{K}\right)$ and, because, even if that was the case, $\widetilde{E}$ need not transform characteristic functions into characteristic functions.

If $j: \Delta \longrightarrow K$ is the inclusion, then $j^{o}: C(K) \longrightarrow C(\Delta)$ is the restriction operator. So, $\widetilde{j^{o}}: B\left(\Sigma_{K}\right) \longrightarrow B\left(\Sigma_{\Delta}\right)$ and it is easy to verify that

$$
\widetilde{j^{o}} \circ \bar{E}=I d_{B\left(\Sigma_{\Delta}\right)}
$$

and that, for every $f, g \in B\left(\Sigma_{\Delta}\right), \bar{E}(f) \bar{E}(g)=\bar{E}(f g)$; so $\widetilde{j^{o}}(\bar{E}(f) \bar{E}(g))=$ $f g$.

Finally we are ready to define $I_{m}=\bar{E} \circ \widetilde{\varphi^{o}} \circ i_{m}(1 \leq m \leq n)$ and $B=T \circ u \circ j^{o}$. Note that

$$
\widetilde{B}=B_{\left.\right|_{B\left(\Sigma_{K}\right)} ^{* *}}=\left(T^{* *} \circ u^{* *} \circ j^{o * *}\right)_{\left.\right|_{B\left(\Sigma_{K}\right)}}=T^{* *} \circ \widetilde{u} \circ \widetilde{j^{o}}
$$

because $\widetilde{j^{o}}\left(B\left(\Sigma_{K}\right)\right) \subset B\left(\Sigma_{\Delta}\right)$.

So, we have,

$$
\begin{gathered}
\mu_{B}\left(\bigcap_{m=1}^{n} A_{m}^{\prime}\right)=\widetilde{B}\left(\prod_{m=1}^{n} I_{m}\left(\chi_{A_{m}}\right)\right)= \\
=T^{* *} \circ \widetilde{u} \circ \widetilde{j^{o}}\left(\prod_{m=1}^{n} \bar{E} \circ \widetilde{\varphi^{o}} \circ i_{m}\left(\chi_{A_{m}}\right)\right)=T^{* *} \circ \widetilde{u} \circ \widetilde{j^{o}}\left(\bar{E} \circ \widetilde{\varphi^{o}}\left(\chi_{A_{1} \times \cdots \times A_{n}}\right)\right)= \\
=T^{* *}\left(\chi_{A_{1} \times \cdots \times A_{n}}\right)=\widetilde{T}\left(\chi_{A_{1} \times \cdots \times A_{n}}\right)=\mu\left(A_{1} \times \cdots \times A_{n}\right) .
\end{gathered}
$$

Since $B=T \circ u \circ j^{o}$ and since $u$ and $j^{o}$ are onto, we get that, for any surjective operator ideal $\mathcal{U}, T \in \mathcal{U}$ if and only if $B \in \mathcal{U}$. In particular, $T$ is weakly compact if and only if $B$ is weakly compact. Moreover, $\mu$ (resp. $\mu_{B}$ ) is $X$-valued if and only if $\widetilde{T}$ (resp. $\widetilde{B}$ ) is $X$-valued ([2, Theorem VI.2.5]), and this proves the last statement of the theorem.

We can prove a similar result for bounded, finitely additive measures defined on $K^{n}$. 
Theorem 2.2. Let $K$ be a metrizable uncountable compact space, $n \in \mathbb{N}$. Then there exist $n$ different linear and multiplicative isometric inclusions $I_{m}: B\left(\Sigma_{K}\right) \hookrightarrow B\left(\Sigma_{K}\right),(1 \leq m \leq n)$ (taking characteristic functions to characteristic functions) such that, for every Banach space $X$ and every operator $T: B\left(\Sigma_{K^{n}}\right) \longrightarrow X$ there exists an operator $B: B\left(\Sigma_{K}\right) \longrightarrow X^{* *}$ so that, for any $g_{1} \otimes \cdots \otimes g_{n} \in \widehat{\otimes}_{\epsilon}^{n} B\left(\Sigma_{K}\right) \subset B\left(\Sigma_{K^{n}}\right)$,

$$
T\left(g_{1} \otimes \cdots \otimes g_{n}\right)=B\left(\prod_{m=1}^{n} I_{m}\left(g_{m}\right)\right) .
$$

If $T$ is weakly compact, then $B$ is weakly compact and $X$-valued.

Therefore, for any bounded finitely additive measure $\mu: \Sigma_{K^{n}} \longrightarrow X$ there exists a bounded finitely additive measure $\mu^{\prime}: \Sigma_{K} \longrightarrow X^{* *}$ so that, for any $\left(A_{1} \times \cdots \times A_{n}\right) \in \Sigma_{K^{n}}$

$$
\mu\left(A_{1} \times \cdots \times A_{n}\right)=\mu^{\prime}\left(\bigcap_{m=1}^{n} A_{m}^{\prime}\right),
$$

where $I_{m}\left(\chi_{A_{m}}\right)=\chi_{A_{m}^{\prime}}$. Moreover, if $\mu$ is strongly additive then $\mu^{\prime}$ is strongly additive and $X$-valued.

Proof. We prove first the result concerning operators. Let $a: B\left(\Sigma_{K^{n}}\right) \hookrightarrow$ $C\left(K^{n}\right)^{* *}$ be the usual isometric injection, $b: C\left(K^{n}\right)^{* *} \hookrightarrow B\left(\Sigma_{K^{n}}\right)^{* *}$ the bitranspose of the injection $C\left(K^{n}\right) \hookrightarrow B\left(\Sigma_{K^{n}}\right)$ and let $k_{X}: X \hookrightarrow X^{* *}$ and $k_{B\left(\Sigma_{K^{n}}\right)}: B\left(\Sigma_{K^{n}}\right) \hookrightarrow B\left(\Sigma_{K^{n}}\right)^{* *}$ be the usual inclusions of spaces into their biduals. Let then $B=T^{* *} \circ b \circ \widetilde{u} \circ \widetilde{j^{o}}$ and $I_{m}=\bar{E} \circ \widetilde{\varphi^{o}} \circ i_{m}(1 \leq m \leq n)$, where all undefined operators are as in Theorem 2.1. Then

$$
\begin{aligned}
& B\left(\prod_{m=1}^{n} I_{m}\left(g_{m}\right)\right)=T^{* *} \circ b \circ \widetilde{u} \circ \widetilde{j^{o}}\left(\prod_{m=1}^{n} \bar{E} \circ \widetilde{\varphi^{o}} \circ i_{m}\left(g_{m}\right)\right)= \\
= & T^{* *} \circ b \circ \widetilde{u}\left(\prod_{m=1}^{n} \widetilde{\varphi^{o}} \circ i_{m}\left(g_{m}\right)\right)=T^{* *} \circ b \circ a\left(g_{1} \otimes \cdots \otimes g_{m}\right)= \\
= & T^{* *} \circ k_{B\left(\Sigma_{K^{n}}\right)}\left(g_{1} \otimes \cdots \otimes g_{m}\right)=T\left(g_{1} \otimes \cdots \otimes g_{m}\right)
\end{aligned}
$$

If $T$ is weakly compact, then clearly $B$ is weakly compact and $X$-valued.

For the result about measures, note that, as we mentioned before, the space of bounded finitely additive measures $b a\left(\Sigma_{K^{n}} ; X\right)$ is isometrically isomorphic to $\mathcal{L}\left(B\left(\Sigma_{K^{n}}\right) ; X\right)$, and similarly $b a\left(\Sigma_{K} ; X\right)$ is isometrically isomorphic to $\mathcal{L}\left(B\left(\Sigma_{K}\right) ; X\right)$. Hence, to prove the result for measures we notice that $I_{m}: B\left(\Sigma_{K}\right) \longrightarrow B\left(\Sigma_{K}\right)$ is linear and multiplicative, and hence it takes characteristic functions into characteristic functions. So, if $T \in \mathcal{L}\left(B\left(\Sigma_{K^{n}}\right) ; X\right)$ is the operator associated to $\mu$ and $B \in \mathcal{L}\left(B\left(\Sigma_{K}\right) ; X\right)$ is the operator related to $T$ by the first part of the theorem, then $\mu^{\prime}$ is the representing measure of $B$.

For the last statement, note that, according to [2, Theorem VI.1.1], an operator $T \in \mathcal{L}(B(\Sigma) ; X)$ is weakly compact if and only if its representing 
measure is strongly additive and that, if $T$ is weakly compact then $B$ is weakly compact.

REMARK 2.3. a) If $\phi: \Sigma_{K} \longrightarrow \Sigma_{S}$ is a boolean algebra morphism, i.e., an application such that, for every $A, B \in \Sigma_{K}, \phi(A \cup B)=\phi(A) \cup \phi(B)$ and $\phi(K \backslash A)=S \backslash \phi(A)$, then $\phi$ induces a linear multiplicative operator $\bar{\phi}: B\left(\Sigma_{K}\right) \longrightarrow B\left(\Sigma_{S}\right)$ given by $\bar{\phi}\left(\chi_{A}\right)=\chi_{\phi(A)} \cdot \bar{\phi}$ has norm $1, \bar{\phi}\left(\chi_{K}\right)=\chi_{S}$ and $\bar{\phi}$ is an isometry if $\phi(A) \neq \emptyset$ whenever $A \neq \emptyset$. Conversely, if $\psi$ : $B\left(\Sigma_{K}\right) \longrightarrow B\left(\Sigma_{S}\right)$ is a linear multiplicative operator and $\psi\left(\chi_{K}\right)=\chi_{S}$ then we can define a boolean algebra morphism $\phi$ such that $\bar{\phi}=\psi$.

If we have a continuous mapping $\varphi: S \longrightarrow K$, then we can always define a boolean algebra morphism ${ }^{o} \varphi: \Sigma_{K} \longrightarrow \Sigma_{S}$ by ${ }^{o} \varphi(A)=\varphi^{-1}(A)$. This in turn gives a linear multiplicative operator ${ }^{o} \varphi: B\left(\Sigma_{K}\right) \longrightarrow B\left(\Sigma_{S}\right)$ and it is easy to check that $\overline{{ }^{o} \varphi}=\widetilde{\varphi^{o}}$.

The morphism

$$
\begin{aligned}
\phi: \Sigma_{K} & \longrightarrow \Sigma_{K} \\
A & \mapsto A^{\prime},
\end{aligned}
$$

where $I_{m}\left(\chi_{A}\right)=\chi_{A^{\prime}}, I_{m}$ being as in Theorem 2.1, is not a boolean algebra morphism, because $\phi(K)=\Delta \neq K$, but it does verify $\phi(A \cup B)=\phi(A) \cup$ $\phi(B)$ and $\phi(A \cap B)=\phi(A) \cap \phi(B)$.

b) If $T: \widehat{\otimes}_{\epsilon}^{n} C(K) \longrightarrow X$ is symmetric, then $\mu: \Sigma_{K^{n}} \longrightarrow X^{* *}$ is symmetric, in the sense that $\mu\left(A_{1} \times \cdots \times A_{n}\right)=\mu\left(A_{\sigma(1)} \times \cdots \times A_{\sigma(n)}\right)$ for any permutation $\sigma$ of $\{1, \ldots, n\}$. It would be nice to have a symmetric version of Theorems 2.1 and 2.2 saying that, if $T$, and hence $\mu$, are symmetric, then we can choose the operators $I_{m}: B\left(\Sigma_{K}\right) \longrightarrow B\left(\Sigma_{K}\right)(1 \leq m \leq n)$ to be all equal. Unfortunately we have not been able to prove it.

\section{REFERENCES}

[1] D. Cohn, Measure Theory, Birkhauser 1980.

[2] J. Diestel and J. J. Uhl, Vector Measures, Mathematical Surveys, No. 15. American Math. Soc., Providence, R.I., 1977.

[3] A. Pelczynski, Linear extensions, linear averaging and application to linear topological classification of spaces of continuous functions, Disser. Math., 58, (1968).

[4] Z. Semadeni, Banach spaces of continuous functions, PNW, 1971.

[5] J. Taskinen, An application of averaging operators to multilinearity, Math. Ann., 297, (1993) 567-572.

[6] I. Villanueva, Operators on the space of continuous functions on the product of compact spaces, preprint.

E-mail address: ignacio_villanueva@mat.ucm.es

Departamento de Análisis Matemático, Facultad de Matemáticas, UniverSIDAD COMPlutense DE MADRID, MAdRID 28040 